\title{
Droplet motion driven by tensotaxis
}

\author{
Jesus Bueno ${ }^{\mathrm{a}, 1}$, Yuri Bazilevs ${ }^{\mathrm{b}}$, Ruben Juanes ${ }^{\mathrm{c}}$, Hector Gomez ${ }^{\mathrm{a}}$ \\ ${ }^{a}$ Departamento de Métodos Matemáticos e de Representación, Universidade da Coruña. Campus de Elviña, 15192, A Coruña, Spain \\ ${ }^{b}$ Department of Structural Engineering, University of California, San Diego. 9500 Gilman Drive, La Jolla, CA 92093, USA \\ ${ }^{c}$ Department of Civil and Environmental Engineering, Massachusetts Institute of Technology. 77 Massachusetts Avenue, Cambridge, MA 02139, USA
}

\begin{abstract}
It is well documented that cells can migrate in response to gradients in stiffness (durotaxis) and gradients in strain (tensotaxis) in the underlying substrate. Understanding the potential physical mechanisms at play during this motion has motivated recent efforts to unravel the role of surface tension in the interaction between droplets and soft solids. Here, we present a multiphysics phase-field model of fluid-solid interaction, which allows us to isolate the effects of strain gradients-something difficult to achieve in experiments. Our high-fidelity numerical simulations in two and three dimensions elucidate the physics of tensotaxis, and show how localized forces in a soft substrate can be used to move and merge droplets deposited on it.
\end{abstract}

\section{(c) 2011 Published by Elsevier Ltd.}

Keywords: Tensotaxis, Soft materials, Fluid-Structure Interaction (FSI), Complex fluids, Navier-Stokes-Korteweg equations (NSK), Isogeometric Analysis (IGA).

\section{Introduction}

Understanding wetting — the affinity of a solid to a fluid — is essential in many natural and engineered processes spanning a wide range of length and time scales, including microfabrication [1, 2], microfluidics [3, 4, 5], and porous media flow applications like oil recovery $[6,7]$ and geologic carbon sequestration [8]. While wetting and spreading of fluids on rigid substrates has been studied extensively [e.g., 9, 10], the interaction of a partially-wetting fluid with a deformable solid substrate has started to receive attention more recently $[11,12,13,14$, $15,16,17]$. In particular, detailed measurements using confocal microscopy have revealed the universal character of the deformation of soft substrates near the contact line [13], and theory and experimental observation have elucidated important aspects of the interaction between droplets and soft substrates. For example, it was recently shown that liquid drops on deformable substrates attract or repel by the so-called inverted Cheerios effect [18], and that droplets move spontaneously on substrates with stiffness variations [19]. The latter phenomenon is reminiscent of durotaxis-cell motion along stiffness gradients [20, 21]. However, while cells tend to move toward stiffer substrates, droplets have been reported to migrate toward softer

\footnotetext{
1e-mail: jesus.bueno@udc.es
}

regions. This observation has rekindled the debate about the role of mechanics in cell durotaxis.

It has also been observed that cells undergo tensotaxis, that is, motion along strain gradients. Tensotaxis has been consistently observed for fibroblasts which migrate toward areas of higher compressive strains [20]. While several mechanistic models have been proposed [22, 23], the understanding of tensotaxis is still very limited. Controlled experimental studies of tensotaxis are particularly challenging because prestraining the substrate is often accompanied by an increase in the substrate stiffness as a result of nonlinear material response [24], thus producing a combination of tensotaxis and durotaxis. A theoretical model that allows isolating the effects of tensotaxis would contribute to a better understanding of the process.

Here, we show that liquid droplets on soft substrates undergo tensotaxis. Droplets move toward areas of higher compressive strains, the same behavior observed in cell migration. Our methodology is based on a theoretical model that describes the coupled interaction of a solid and a fluid with liquid and gaseous phases. We solve the governing equations by means of high-fidelity numerical simulations, and show that tensotaxis enables the motion and merging of droplets. The migration patterns depend on the solid deformation globally, thus pointing to the usefulness of a computational approach to help elucidate the physics of tensotaxis. 


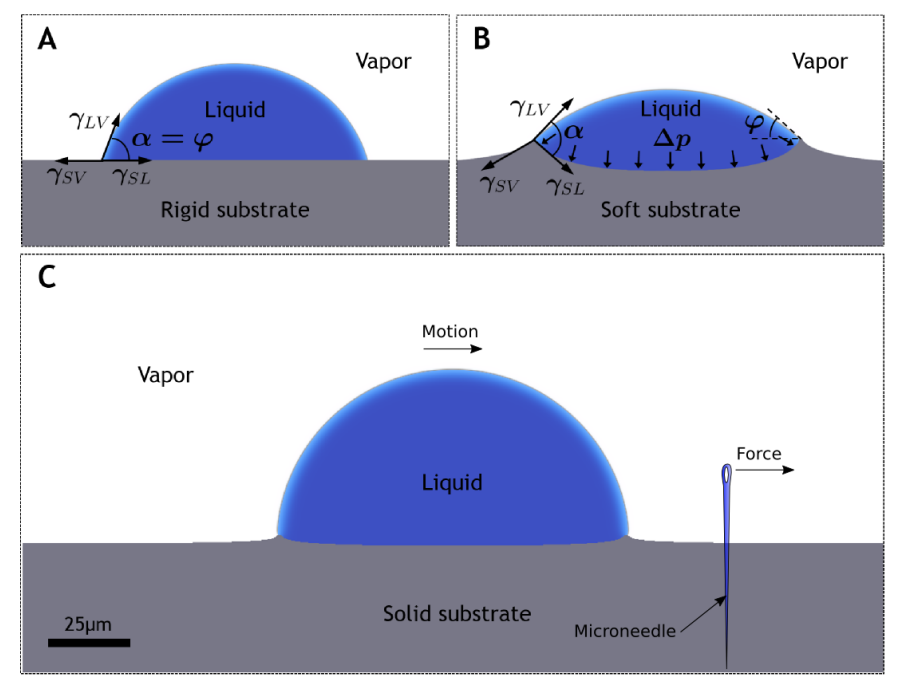

Figure 1. Droplet motion driven by tensotaxis. (A) Liquid droplet (blue) deposited on a rigid substrate (gray). The surface tensions at the contact line $\gamma_{L V}$, $\gamma_{S V}$ and $\gamma_{S L}$ are represented with arrows. (B) Liquid droplet on a soft substrate. The solid is deformed under the combined action of the surface tensions and the internal Laplace pressure $\Delta p$. The plot shows that the static contact angle $\alpha$ and the apparent contact angle $\varphi$ are different when the solid is deformable. (C) Tensotaxis can be triggered by inserting a microneedle in the substrate and moving it toward the droplet or away from the droplet. The deformation produced by the droplet has been computed using the model presented in Section 2.1. The computational domain $\Omega=[0,1.0] \times[0,0.5]$ is discretized with a uniform mesh of $512 \times 256 C^{1}$-quadratic elements. We have adopted the parameters $v=0.45$, $\widehat{\mu}=1 / 1024, \widehat{\gamma}=1 / 512, \widehat{E}=0.1$, and $\widehat{\theta}=0.39$. Neither gravity nor other external forces were considered in this simulation, i.e, $\left|\widehat{f}^{s}\right|=0.0$.

\section{Model of droplet tensotaxis}

The static configuration that droplets adopt on rigid, flat substrates is governed by the Young-Dupré equation, $\gamma_{S L}+$ $\gamma_{L V} \cos \alpha=\gamma_{S V}$, where $\alpha$ is the static contact angle, and $\gamma_{L V}$, $\gamma_{S V}$ and $\gamma_{S L}$ denote the interfacial tension at the liquid-vapor, solid-vapor and solid-liquid interfaces, respectively [25] [Fig. 1(A) When the substrate is soft, elastocapillary forces create a dimple below the droplet and a ridge at the contact line. This produces a rotation of the liquid-vapor interface [13], which after deformation is oriented at an apparent contact angle $\varphi<\alpha$ with respect to the horizontal [Fig. 1(B)]. Elastocapillary forces are relevant when the elastocapillary length $l_{e c}=\gamma_{L V} / E^{s}\left(E^{s}\right.$ is the Young modulus of the solid) is comparable to the droplet radius. For values of $l_{e c}$ much smaller than the droplet radius, the deformation of the solid is negligible. Most theoretical efforts to understand the interaction of droplets and deformable substrates are based on thin film descriptions of the fluid problem and linear elastic solids $[12,18]$. The approaches are variational and allow computing a minimum-energy configuration. Here, we study the interaction between droplets and a soft solid by developing a three-dimensional model that couples the nonlinear dynamics of a solid with a fluid composed of a liquid and a gaseous phase. We obtain numerical solutions to the proposed equations with a computational method based on isogeometric analysis [26]. Using this computational approach, we show that droplet tensotaxis emerges in a system that mimics the one employed in cell locomotion experiments [20]. A microneedle is inserted into the substrate and exerts a force either toward the droplet or away from the droplet [Fig. 1(C)]. The droplet moves in the direction of the force applied by the needle.

\subsection{Methods}

We develop a fluid-structure interaction model that couples a Saint Venant-Kirchhoff solid with a complex fluid. Our choice of a Saint Venant-Kirchhoff model for the solid allows us to consider geometric nonlinearities with a linear material response. As a consequence, the strains introduced in the substrate to trigger tensotaxis do not alter the stiffness of the solid, avoiding a situation with simultaneous tensotaxis and durotaxis. The fluid is governed by the Navier-Stokes-Korteweg (NSK) equations, a phase-field theory that allows for the stable coexistence of a liquid and a gaseous phase.

\section{Solid mechanics equations}

The solid dynamics is described by the Lagrangian form of the momentum balance equation

$$
\left.\rho_{0}^{s} \frac{\partial^{2} \boldsymbol{u}}{\partial t^{2}}\right|_{\boldsymbol{X}}=\nabla_{\boldsymbol{X}} \cdot \boldsymbol{P}+\rho_{0}^{s} \boldsymbol{f}^{s},
$$

where $\nabla_{X}$ is the gradient with respect to the material coordinates $\boldsymbol{X}$ and $\left.\right|_{X}$ indicates that the time derivative is taken by holding $\boldsymbol{X}$ fixed; $\boldsymbol{u}$ is the solid displacement and $\rho_{0}^{s}$ is the mass density in the initial configuration; $\boldsymbol{f}^{s}$ represents body forces per unit mass, and $\boldsymbol{P}$ is the first Piola-Kirchhoff stress tensor. The Saint Venant-Kirchhoff model is described by the stored elastic energy density [27, 28]

$$
W=\frac{\lambda^{s}}{2}(\operatorname{tr}(\boldsymbol{E}))^{2}+\mu^{s} \operatorname{tr}\left(\boldsymbol{E}^{2}\right),
$$

Here, $\operatorname{tr}(\cdot)$ denotes the trace operator whereas $\lambda^{s}$ and $\mu^{s}$ are the first and second Lamé parameters, which can be written as a A)]function of the Young modulus $E^{s}$ and the Poisson ratio $v: \lambda^{s}=$ $v E^{s} /((1+v)(1-2 v))$ and $\mu^{s}=E^{s} /(2(1+v))$. The GreenLagrange strain tensor is defined by $\boldsymbol{E}=(\boldsymbol{C}-\boldsymbol{I}) / 2$, where $\boldsymbol{I}$ denotes the identity tensor and $\boldsymbol{C}=\boldsymbol{F}^{T} \boldsymbol{F}$ is the Cauchy-Green deformation tensor. Here, $\boldsymbol{F}$ denotes the deformation gradient, i.e., $\boldsymbol{F}=\boldsymbol{I}+\nabla_{X} \boldsymbol{u}$. The second Piola-Kirchhoff stress tensor can be computed from $W$ as $\boldsymbol{S}=\partial W / \partial \boldsymbol{E}$ while the first PiolaKirchhoff stress tensor is obtained by $\boldsymbol{P}=\boldsymbol{F S}$. The Cauchy stress tensor in the solid is given by $\boldsymbol{\sigma}^{s}=J^{-1} \boldsymbol{F} \boldsymbol{S} \boldsymbol{F}^{T}=J^{-1} \boldsymbol{P} \boldsymbol{F}^{T}$, where $J=\operatorname{det}(\boldsymbol{F})$.

\section{Fluid mechanics equations}

We use the isothermal Navier-Stokes-Korteweg (NSK) equations to describe the fluid dynamics. The NSK equations account for mass and momentum conservation. They describe single-component two-phase flow and naturally allow for phase transformations, which can happen spontaneously due to pressure and/or temperature variations. The multiphase nature of the flow is treated using the phase-field method. Phase-field models, also known as diffuse-interface models, represent an alternative to sharp-interface models, in which interfaces are 
define an order parameter, or phase-field, that varies smoothly over the entire computational domain and acts as a marker for the location of the different phases [29]. In the NSK theory, the fluid density itself is the phase-field that identifies the liquid and vapor phases. Phase-field models have been successfully used in many fields [e.g., 30, 31, 32, 33, 34, 35], including the description of partial wetting [36, 37]. The phase-field modeling permits, in our case, a unified and efficient computational treatment of the coupled multiphysics problem.

In the Eulerian description, the isothermal NSK equations are given by

$$
\begin{aligned}
& \frac{\partial \rho}{\partial t}+\nabla \cdot(\rho \boldsymbol{v})=0, \\
& \frac{\partial(\rho \boldsymbol{v})}{\partial t}+\nabla \cdot(\rho \boldsymbol{v} \otimes \boldsymbol{v})-\nabla \cdot \boldsymbol{\sigma}^{f}=0,
\end{aligned}
$$

where $\rho$ is the density, $\boldsymbol{v}$ is the velocity vector, $\boldsymbol{\sigma}^{f}$ is the fluid stress tensor and $\otimes$ denotes the outer vector product. The Cauchy stress tensor for the fluid is defined as $\boldsymbol{\sigma}^{f}=\boldsymbol{\tau}-p \boldsymbol{I}+\boldsymbol{\boldsymbol { S }}$, where $\boldsymbol{\tau}$ is the viscous stress tensor, $p$ denotes the pressure, and $\boldsymbol{s}$ is the so-called Korteweg tensor. As we consider Newtonian fluids, the viscous stress tensor is given by

$$
\boldsymbol{\tau}=\bar{\mu}\left(\nabla \boldsymbol{v}+\nabla^{T} \boldsymbol{v}\right)+\bar{\lambda} \nabla \cdot \boldsymbol{v I},
$$

where $\bar{\mu}$ and $\bar{\lambda}$ are the viscosity coefficients, which are assumed to be related through the Stokes hypothesis, that is, $\bar{\lambda}=-2 \bar{\mu} / 3$. The Korteweg tensor [38, 39] is defined by

$$
\boldsymbol{\varsigma}=\lambda\left(\rho \Delta \rho+\frac{1}{2}|\nabla \rho|^{2}\right) \boldsymbol{I}-\lambda \nabla \rho \otimes \nabla \rho,
$$

where $|\cdot|$ denotes the Euclidean norm of a vector, and $\lambda>0$ is the capillarity coefficient. The Korteweg tensor results in the capillary forces that are withstood by the liquid-vapor interfaces.

We use the Helmholtz free-energy of a van der Waals fluid $[40,41]$ to allow for stable coexistence of liquid and gas phases. Using the Helmholtz free-energy and standard thermodynamics [41], we obtain the van der Waals equation, which gives the pressure $p$ in terms of density and temperature $\theta$, i.e.,

$$
p=R b\left(\frac{\rho \theta}{b-\rho}\right)-a \rho^{2} .
$$

Here, $a$ and $b$ are positive constants and $R$ is the specific gas constant.

Since the NSK system includes a third-order spatial derivative of the fluid density in the linear momentum balance equation, the classical solid-wall boundary conditions are insufficient to render a well-posed boundary value problem. Therefore, we additionally impose the boundary condition $\nabla \rho \cdot \boldsymbol{n}^{f}=$ $|\nabla \rho| \cos \alpha$, where $\boldsymbol{n}^{f}$ denotes the unit outward normal to the fluid boundary, and $\alpha$ is the contact angle between the liquid-vapor interface and the solid surface, measured in the liquid phase (see Fig. 1). This boundary condition prescribes the contact angle $\alpha$ at the fluid-structure interface, while the apparent contact angle $\varphi$ is determined as part of the solution to the coupled equations as a result of the substrate deformation.
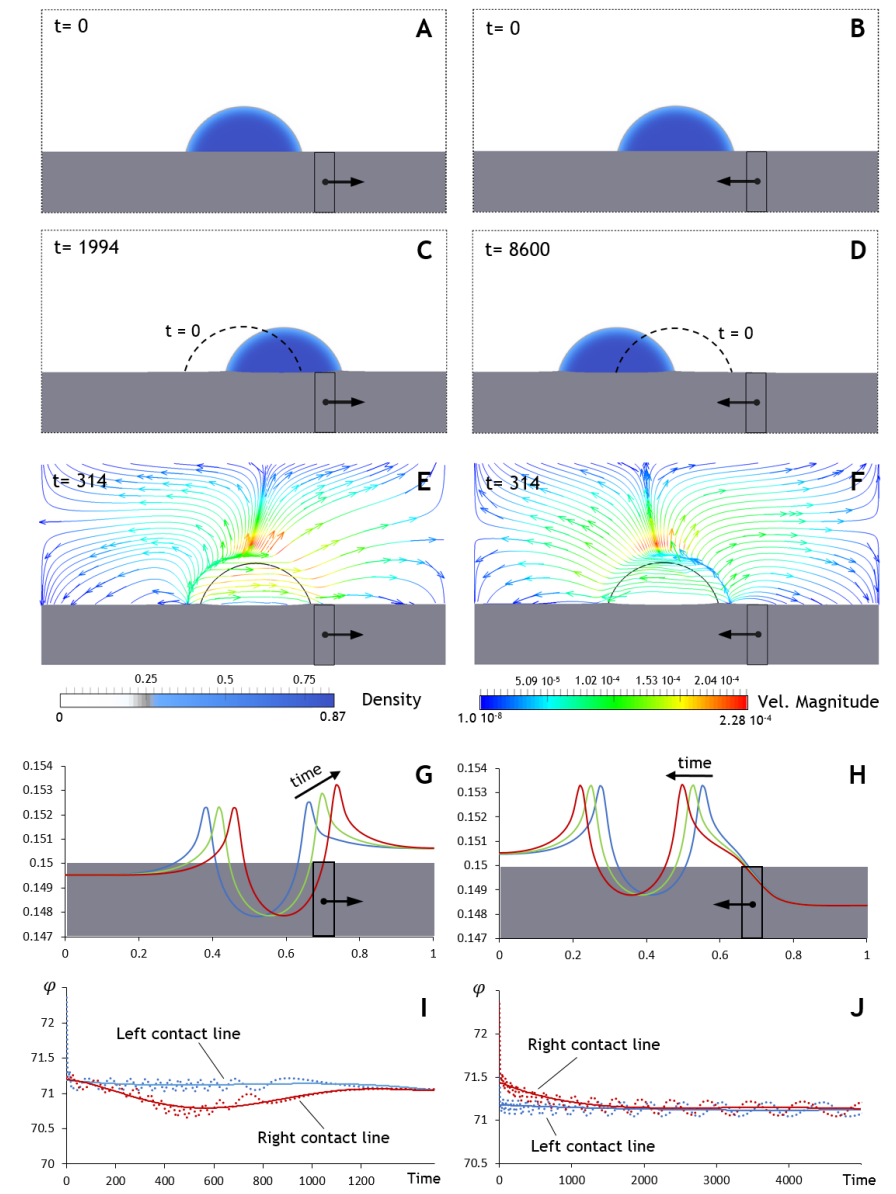

Figure 2. Mechanism of tensotaxis. Left panels refer to the case in which the applied force pulls the droplet. Right panels refer to case in which the applied force pushes the droplet. (A) and (B) Initial configuration of a droplet on a deformable substrate. We apply a force per unit mass that points away from the droplet (A) and toward the droplet (B) in the marked rectangular region. (C) and (D) The droplet moves in the direction of the applied force. The dashed black line represents the position of the droplet at the initial time. (E) and (F) Streamlines of the fluid velocity colored with the velocity magnitude. The droplet is represented by a black, solid line. (G) Vertical displacements at the fluid-solid interface at three different dimensionless times, $t=235, t=471$ and $t=1884$. (H) Vertical displacements at the fluid-solid interface at time $t=1962, t=3924$ and $t=7848$. (I) and (J) Time evolution of the apparent contact angles at the left (blue dashed line) and right (red dashed line) contact lines of the droplet. Trend lines are ploted using a blue and red solid lines, respectively. The difference in apparent contact angles between the two contact lines is responsible for the motion of the droplet. The computational domain is the rectangle $\Omega=[0,1.0] \times[0,0.5]$, which is discretized with a uniform mesh of $128 \times 64 C^{1}$-quadratic elements. On the left, right and lower boundaries of the computational domain, we impose zero velocity in normal direction. On the upper boundary, zero velocity is imposed in both directions. The static contact angle is $\alpha=75^{\circ}$. We have used the parameters $v=0.45, \widehat{\mu}=1 / 256, \widehat{\gamma}=1 / 64$, $\widehat{E}=0.7554$, and $\widehat{\theta}=0.39$. The magnitude of the force applied on the substrate is $\left|\widehat{f}^{s}\right|=0.16215$. 


\section{Coupled problem}

The differential equations governing the solid and fluid motions must be satisfied simultaneously. These equations are coupled at the fluid-solid interface through compatibility conditions. We impose kinematic compatibility $(\boldsymbol{v}=\partial \boldsymbol{u} / \partial t)$ and traction balance $\left(\boldsymbol{\sigma}^{f} \boldsymbol{n}^{f}-\boldsymbol{\sigma}^{s} \boldsymbol{n}^{f}=0\right)$.

\section{Computational method}

Our computational approach is similar to those presented in [42, 43]. We solve the coupled system composed by Eq. (1) and Eq. (3) subject to the kinematic compatibility and traction balance constraints. Equation (1) is solved in the reference (undeformed) configuration of the solid domain. Equation (3) is solved in the spatial domain occupied by the fluid, which changes over time. This requires the use of geometrically flexible algorithms, such as the finite element method. Here, we use isogeometric analysis, which is a spline-based finite-elementlike method that combines geometric flexibility with smooth basis functions [44, 26]. The use of smooth basis functions allows for a direct discretization of higher-order partial differential equations such as the NSK equation. To enable the use of classical finite-difference-type methods for time integration, we recast the NSK equations in an arbitrary Lagrangian Eulerian (ALE) formulation:

$$
\begin{aligned}
& \left.\frac{\partial \rho}{\partial t}\right|_{\widehat{x}}+(\boldsymbol{v}-\widehat{\boldsymbol{v}}) \cdot \nabla \rho+\rho \nabla \cdot \boldsymbol{v}=0 \\
& \left.\rho \frac{\partial \boldsymbol{v}}{\partial t}\right|_{\widehat{x}}+\rho(\boldsymbol{v}-\widehat{\boldsymbol{v}}) \cdot \nabla \boldsymbol{v}-\nabla \cdot \boldsymbol{\sigma}^{f}=0 .
\end{aligned}
$$

Here, $\widehat{\boldsymbol{v}}$ is the fluid domain velocity and $\widehat{\boldsymbol{x}}$ is a coordinate in a reference domain that is used for computational purposes. Equations (1) and (7) can then be written in variational form and discretized in space using isogeometric analysis. We use the generalized $-\alpha$ method [45] as a time integration scheme. The nonlinear system of equations is solved using a NewtonRaphson iteration procedure, which leads to a two-stage predictormulticorrector algorithm. The resulting linear system is solved using a preconditioned GMRES method.

We express the problem in non-dimensional form by rescaling length, time, mass and temperature by $L_{0}, L_{0} / \sqrt{a b}, b L_{0}^{3}$ and $\theta_{c}$, respectively, where $L_{0}=1$ is a length scale of the computational domain and $\theta_{c}=8 a b /(27 R)$ is the so-called critical temperature. Using this non-dimensionalization, the problem is characterized by the following dimensionless numbers,

$$
\begin{aligned}
\widehat{\gamma} & =\frac{\sqrt{\lambda / a}}{L_{0}} \quad \text { (dimensionless surface tension), } \\
\widehat{\mu} & =\frac{\bar{\mu}}{L_{0} b \sqrt{a b}} \quad \text { (dimensionless viscosity), } \\
\widehat{\theta} & =\frac{\theta}{\theta_{c}} \quad \text { (dimensionless temperature) } \\
v & (\text { Poisson ratio), } \\
\widehat{E} & =\frac{E^{s}}{\rho_{0}^{s} a b} \quad \text { (dimensionless Young modulus), } \\
\widehat{\boldsymbol{f}}^{s} & =\frac{\boldsymbol{f}^{s}}{a b / L_{0}} \quad \text { (dimensionless body force). }
\end{aligned}
$$
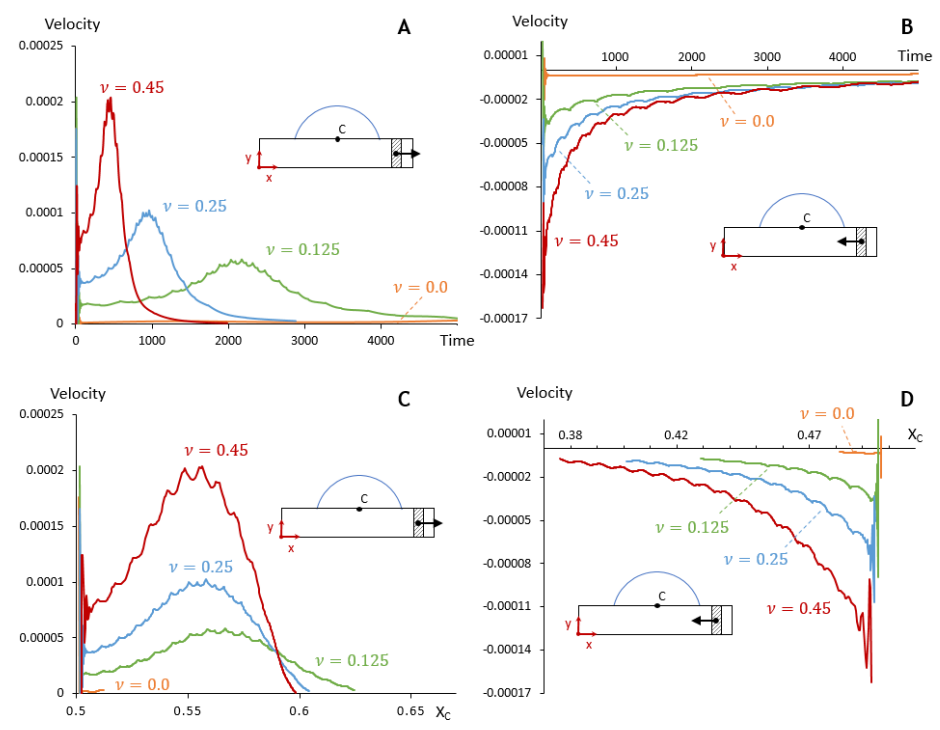

Figure 3. Pulling and pushing the droplet. Left panels refer to the case in which the applied force pulls the droplet. Right panels refer to case in which the applied force pushes the droplet. (A) and (B) Droplet velocity with respect to time for different Poisson's ratios $v$. The velocity of the droplet is computed as the average of the contact line velocities. When pulling, the droplet initially accelerates and then decelerates as it passes through the localized applied force, and is eventually trapped (A). When pushing, the magnitude of the velocity is monotonically decreasing as the influence of the applied force decreases as the droplet moves away from the force (B). (C) and (D) Droplet velocity with respect to the position of the droplet center $X_{c}$, which is assumed to be at the midpoint between the contact line positions at each time step. In all cases the velocity is reduced as the Poisson ratio drops, and for $v=0$ the applied force induces no droplet motion.

In all our computations, the values adopted for the dimensionless surface tension and viscosity were chosen according to the upscaling method proposed in [46], which relates these parameters to the computational mesh.

\section{Results}

We begin by performing numerical simulations of droplet motion that mimic the experiments conducted for cells [Fig. 1(C)]. In our computations, the effect of the needle is modeled as a horizontal force per unit mass applied on a localized area [Figs. 2(A)-(B)]. Our simulations show that the droplet moves in the direction of the force [Figs. 2(C)-(D)]. The coupling between solid and fluid elicits flow of the gas phase surrounding the droplet [Figs. 2(E)-(F)]. The applied load produces vertical displacements in the solid, as it would be expected in a material with a nonzero Poisson ratio [Figs. $2(\mathrm{G})-(\mathrm{H})]$. These vertical displacements are opposite in sign at each side of the load. The droplet's excess pressure produces a depression of the substrate, while the localized force at the contact line pulls up the solid. Away from the droplet the substrate acquires a flat shape, but the vertical displacement is different at both sides of the droplet and is controlled by the external force applied to the substrate: positive where the load induces compressive stresses, and negative on the side where the load induces tensile stresses. The difference in vertical displacements at either side of the droplet 
produces a rotation of the droplet that drives motion. This can be understood with a force balance in the deformed contact line, which is rotated due to the solid compliance [13]. The solid deformation produces different apparent contact angles at the two contact lines, leading to an unbalanced horizontal force similar to that present when the wettability of the substrate is different at either contact line [Figs. 2(I)-(J)]. The tensotaxis mechanism is independent of gravity, which is negligible at this scale.

Further insight into the mechanics of tensotaxis is gained by plotting the droplet velocity with respect to time and space (Fig. 3). It is apparent from these simulations that the behavior is remarkably different depending on the direction of the applied force. Pulling induces increasing velocities of the droplet as the contact line approaches the region of the applied force, but ultimately trap the droplet in the loaded area [Figs. 3(A) and $(\mathrm{C})]$. In contrast, pushing repels the droplet monotonically, albeit with a time-decreasing velocity [Figs. 3(B) and (D)]. In all cases, the droplet velocity is smaller as the Poisson ratio decreases - indeed, the droplet remains immobile if the Poisson ratio is zero. All these observations are consistent with the proposed mechanism of droplet tensotaxis.

To more faithfully represent the physical reality we also carried out $3 \mathrm{D}$ simulations of tensotaxis. The $3 \mathrm{D}$ analogue of the needle experiment in Fig. 1(C) is shown in Fig. 4. A horizontal force applied at the center of the substrate drives droplet motion in the direction of the force [Fig. 4(B)]. A cross section of the system through the (diagonal) axis of symmetry shows that the substrate deformation is consistent with the picture that emerges from the 2D simulations [Fig. 4(C)].

The investigation of tensotaxis behavior in the presence of multiple droplets is revealing. The presence of multiple droplets increases the complexity of the problem, as we must consider their mechanical interactions through the deformable substrate, as well as through the fluid domain. We simulate this interaction in a system with two droplets of different size, each "pushed" toward the other by the action of a localized force [Fig. 5(A)]. As the droplets approach each other, they eventually coalesce [Fig. 5(B)] and quickly readjust into a single droplet, trapped by the two applied forces of opposite sign [Fig. 5(C)]. These predictions illustrate the ability of the phase-field methodology to simulate complex scenarios of tensotaxis in $3 \mathrm{D}$, in which the coupling between surface tension forces and substrate deformation lead to droplet motion, coalescence and trapping.

\section{Conclusions}

Tensotaxis, or motion driven by strain gradients, has been reproducibly observed for several cell types, but the mechanisms that control the process remain unknown. Here, we have shown that simple liquid droplets on soft substrates also undergo tensotaxis. We have used a nonlinear coupled model of fluid-structure interaction to elucidate the physics of droplet tensotaxis. Our results indicate that droplet tensotaxis is controlled by the global deformation of the solid, making the migration pattern sensitive to boundary conditions and additional
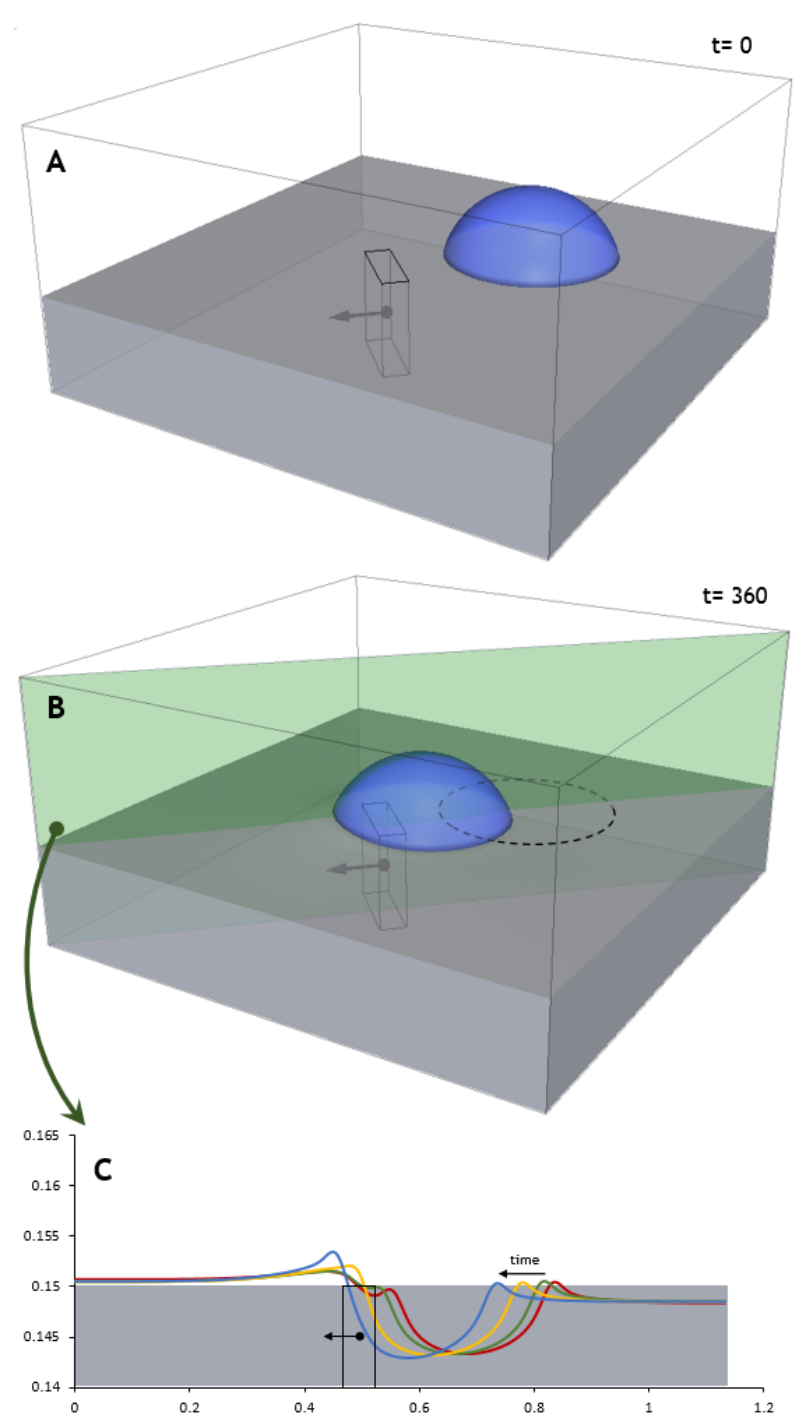

Figure 4. Three-dimensional droplet motion triggered by tensotaxis. (A) Initial configuration of the numerical experiment. A liquid droplet is deposited on a deformable substrate. A horizontal force is applied at the center of the substrate. (B) The droplet moves in the direction of the applied force. The black, dashed line on the surface of the substrate indicates the initial position of the droplet. (C) Vertical displacement of the solid-liquid interface at the diagonal plane [green color in panel (B)] at different times, $t=63$ (red), $t=125$ (green), $t=188$ (yellow), and $t=251$ (blue). The computational domain $\Omega=[0,0.8] \times$ $[0,0.8] \times[0,0.4]$ is discretized with $80 \times 80 \times 40 C^{1}$-quadratic elements. On the upper boundary, we prescribe zero velocity in the horizontal and vertical directions. On the lateral and lower boundaries, zero velocity is imposed in normal direction. We have adopted $v=0.45, \widehat{\mu}=1 / 200, \widehat{\gamma}=1 / 50, \widehat{E}=0.7554$, and $\widehat{\theta}=0.39$. The load that triggers droplet motion is a body force per unit mass of value $\left|\widehat{\boldsymbol{f}}^{s}\right|=1.376$. The static contact angle is $\alpha=75^{\circ}$. 

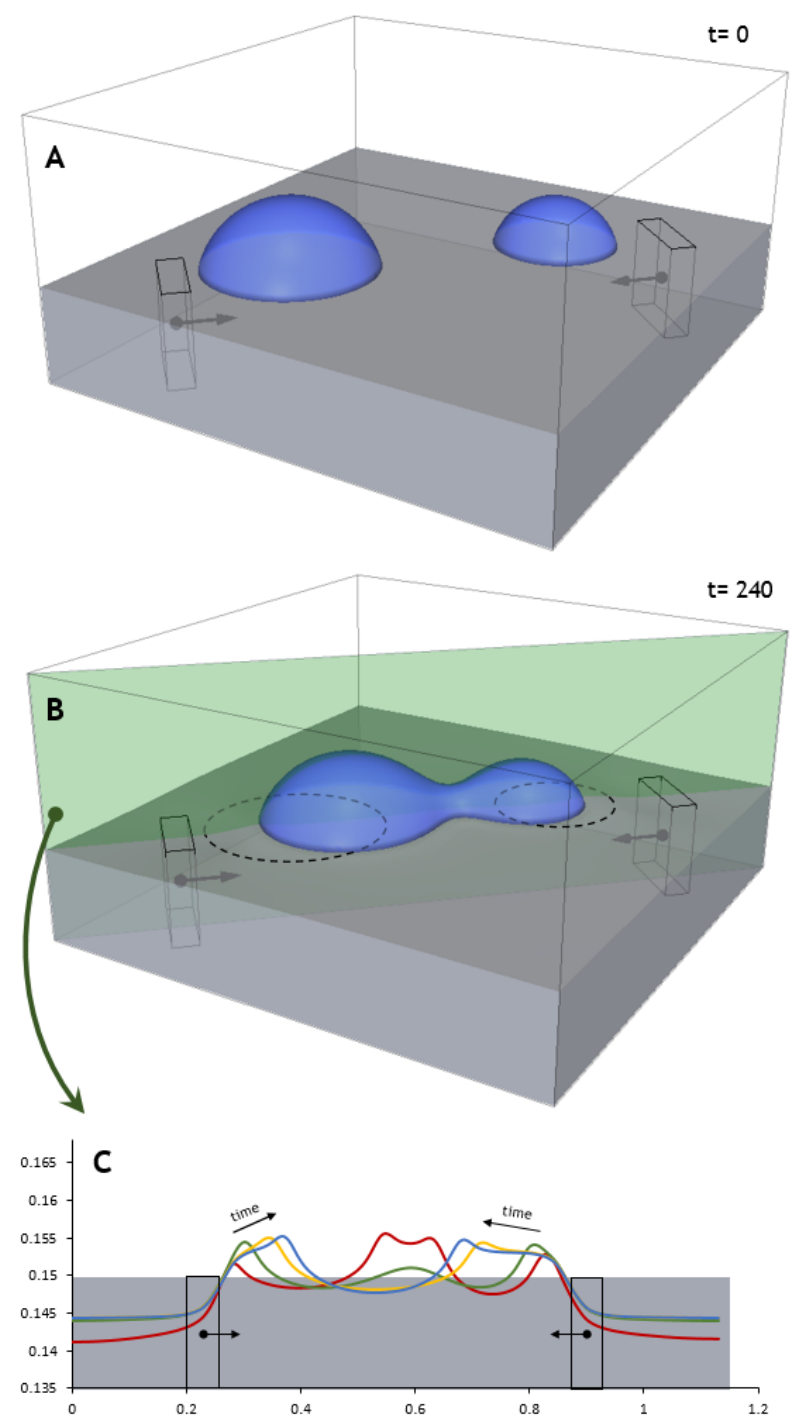

Figure 5. Droplet coalescence induced by tensotaxis. (A) Setup of the numerical experiment. Two forces of the same magnitude are applied on the substrate, pushing the droplets towards the center of the domain. (B) When the droplets are sufficiently close, capillary forces promote coalescence of the two droplets. The black, dashed line on the surface of the substrate represents the initial position of the droplets. (C) Vertical displacement at the solid-fluid interface at different times, $t=66$ (red), $t=240$ (green), $t=267$ (yellow), and $t=314$ (blue). The computational domain is the box $\Omega=[0,0.8] \times[0,0.8] \times[0,0.4]$, which is comprised of $80 \times 80 \times 40 C^{1}$-quadratic elements. On the lateral and lower boundaries, we impose zero velocity in normal direction. On the upper boundary, zero velocity is prescribed in the horizontal and vertical directions. We have adopted $v=0.45, \widehat{\mu}=1 / 200, \widehat{\gamma}=1 / 50, \widehat{E}=0.7554$, and $\widehat{\theta}=0.39$. The load that triggers droplet motion is a body force per unit mass of value $\left|\widehat{\boldsymbol{f}}^{s}\right|=2.7519$. The static contact angle is $\alpha=75^{\circ}$. external loads. Although the droplet always moves in the direction of the force-as observed in cell locomotion experimentsour results reveal the symmetry-breaking depending on the nature of the applied force. Upon the action of pulling forces, a droplet first accelerates but is then trapped as it traverses the localized force. The action of pushing forces, in contrast, leads to monotonic motion of the droplet with decaying velocity. The computational model suggests that droplet tensotaxis occurs for arbitrary values of the applied force, while cell tensotaxis has been only observed for loads that are larger than a threshold value. This might be a consequence of active contractile forces exerted by the cell on the substrate, which are not considered in droplet tensotaxis. Our computational model also reveals the key role of Poisson's ratio in tensotaxis, and the ability of localized forces to induce droplet coalescence. These observations from computational modeling allow us to gain insight into the mechanisms that govern tensotaxis, and may suggest new experimental studies for cell migration.

\section{Acknowledgments}

HG was partially supported by the European Research Council through the FP7 Ideas Starting Grant Program (Contract \#307201). HG and JB were partially supported by Xunta de Galicia, co-financed with FEDER funds. JB is grateful to the Ph.D student grant UDC-Inditex for the financial support during his visit at the University of California, San Diego, where part of this work was accomplished. RJ acknowledges support from the U.S. Department of Energy through a DOE Mathematical Multifaceted Integrated Capability Center Award (Grant No. DE-SC0009286).

\section{References}

[1] M. Srinivasarao, D. Collings, A. Philips, S. Patel, Three-dimensionally ordered array of air bubbles in a polymer film, Science 292 (5514) (2001) 79-83.

[2] D. B. Weibel, W. R. DiLuzio, G. M. Whitesides, Microfabrication meets microbiology, Nature Reviews Microbiology 5 (2007) 209-218.

[3] P. Tabeling, S. Chen, Introduction to Microfluidics, Oxford University Press, 2005.

[4] T. M. Squires, S. R. Quake, Microfluidics: Fluid physics at the nanoliter scale, Reviews of Modern Physics 77 (3) (2005) 977-1026.

[5] B. Zhao, C. W. MacMinn, R. Juanes, Wettability control on multiphase flow in patterned microfluidics, Proceedings of the National Academy of Sciences 113 (37) (2016) 10251-10256.

[6] D. C. Standnes, T. Austad, Wettability alteration in carbonates: Interaction between cationic surfactant and carboxylates as a key factor in wettability alteration from oil-wet to water-wet conditions, Colloids Surf. A 216 (2003) 243-259.

[7] M. Trojer, M. L. Szulczewski, R. Juanes, Stabilizing fluid-fluid displacements in porous media through wettability alteration, Physical Review Applied 3 (2015) 054008.

[8] P. Chiquet, D. Broseta, S. Thibeau, Wettability alteration of caprock minerals by carbon dioxide, Geofluids 7 (2) (2007) 112-122.

[9] P. G. de Gennes, Wetting: statics and dynamics, Reviews of Modern Physics 57 (1985) 827-863.

[10] D. Bonn, J. Eggers, J. Indekeu, J. Meunier, E. Rolley, Wetting and spreading, Review of Modern Physics 81 (2009) 739-805.

[11] C. Duprat, S. Protiere, A. Y. Beebe, H. A. Stone, Wetting of flexible fibre arrays, Nature 482 (7386) (2012) 510-513.

[12] R. W. Style, E. R. Dufresne, Static wetting on deformable substrates, from liquids to soft solids, Soft Matter 8 (27) (2012) 7177-7184. 
[13] R. W. Style, R. Boltyanskiy, Y. Che, J. S. Wettlaufer, L. A. Wilen, E. R. Dufresne, Universal deformation of soft substrates near a contact line and the direct measurement of solid surface stresses, Physical Review Letters 110 (2013) 066103.

[14] T. Kajiya, A. Daerr, T. Narita, L. Royon, F. Lequeux, L. Limat, Advancing liquid contact line on visco-elastic gel substrates: stick-slip vs. continuous motions, Soft Matter 9 (2013) 454-461.

[15] J. B. Bostwick, M. Shearer, K. E. Daniels, Elastocapillary deformations on partially-wetting substrates: rival contact-line models, Soft Matter 10 (2014) 7361-7369.

[16] S. Karpitschka, S. Das, M. Van Gorcum, H. Perrin, B. Andreotti, J. Snoeijer, Droplets move over viscoelastic substrates by surfing a ridge, Nature Communications 6 (2015) 7891.

[17] B. Andreotti, O. Bäumchen, F. Boulogne, K. E. Daniels, E. R. Dufresne, H. Perrin, T. Salez, J. H. Snoeijer, R. W. Style, Solid capillarity: when and how does surface tension deform soft solids?, Soft Matter 12 (12) (2016) 2993-2996.

[18] S. Karpitschka, A. Pandey, L. A. Lubbers, J. H. Weijs, L. Botto, S. Das, B. Andreotti, J. H. Snoeijer, Liquid drops attract or repel by the inverted cheerios effect, Proceedings of the National Academy of Sciences 113 (27) (2016) 7403-7407.

[19] R. W. Style, Y. Che, S. J. Park, B. M. Weon, J. H. Je, C. Hyland, G. K. German, M. P. Power, L. A. Wilen, J. S. Wettlaufer, et al., Patterning droplets with durotaxis, Proceedings of the National Academy of Sciences 110 (31) (2013) 12541-12544.

[20] C.-M. Lo, H.-B. Wang, M. Dembo, Y.-1. Wang, Cell movement is guided by the rigidity of the substrate, Biophysical Journal 79 (1) (2000) 144152.

[21] R. Sunyer, V. Conte, J. Escribano, A. Elosegui-Artola, A. Labernadie, L. Valon, D. Navajas, J. M. García-Aznar, J. J. Muñoz, P. Roca-Cusachs, $\mathrm{X}$. Trepat, Collective cell durotaxis emerges from long-range intercellular force transmission 353 (6304) (2016) 1157-1161.

[22] I. B. Bischofs, U. S. Schwarz, Cell organization in soft media due to active mechanosensing, Proceedings of the National Academy of Sciences 100 (16) (2003) 9274-9279.

[23] P. Moreo, J. M. García-Aznar, M. Doblaré, Modeling mechanosensing and its effect on the migration and proliferation of adherent cells, Acta Biomaterialia 4 (3) (2008) 613-621.

[24] S.-L. Lin, J.-C. Yang, K.-N. Ho, C.-H. Wang, C.-W. Yeh, H.-M. Huang, Effects of compressive residual stress on the morphologic changes of fibroblasts, Medical \& Biological Engineering \& Computing 47 (12) (2009) 1273-1279.

[25] P.-G. De Gennes, F. Brochard-Wyart, D. Quéré, Capillarity and Wetting Phenomena: Drops, Bubbles, Pearls, Waves, Springer Science \& Business Media, 2004

[26] J. Cottrell, T. Hughes, Y. Bazilevs, Isogeometric Analysis: Toward Integration of CAD and FEA, Wiley, 2009.

[27] J. E. Marsden, T. J. R. Hughes, Mathematical Foundations of Elasticity, Prentice-Hall, Englewood Cliffs, NJ, 1983, reprinted with corrections, Dover, New York, 1994.

[28] Y. Bazilevs, K. Takizawa, T. Tezduyar, Computational Fluid-Structure Interaction. Methods and Applications, Wiley, 2013.

[29] H. Gomez, K. van der Zee, Encyclopedia of Computational Mechanics. Phase Field Models and Methods, John Wiley \& Sons, Ltd, 2016.

[30] G. Caginalp, Stefan and hele-shaw type models as asymptotic limits of the phase-field equations, Physical Review A 39 (1989) 5887-5896.

[31] D. M. Anderson, G. B. McFadden, A. A. Wheeler, Diffuse-interface methods in fluid mechanics, Annual Review of Fluid Mechanics 30 (1998) 139-165.

[32] J. Jeong, N. Goldenfeld, J. Dantzig, Phase field model for threedimensional dendritic growth with fluid flow, Physical Review E 64 (2001) 041602

[33] W. J. Boettinger, J. A. Warren, C. Beckermann, A. Karma, Phase-field simulation of solidification, Annual Review of Materials Research 32 (2002) 163-194.

[34] T. Biben, K. Kassner, C. Misbah, Phase-field approach to threedimensional vesicle dynamics, Physical Review E 72 (2005) 041921.

[35] H. Gomez, L. Cueto-Felgueroso, R. Juanes, Three-dimensional simulation of unstable gravity-driven infiltration of water into a porous medium, Journal of Computational Physics 238 (2013) 217-239.

[36] L. Cueto-Felgueroso, R. Juanes, Macroscopic phase-field modeling of partial wetting: bubbles in a capillary tube, Physical Review Letters 108 (2012) 144502

[37] A. Alizadeh Pahlavan, L. Cueto-Felgueroso, R. Juanes, Thin films in partial wetting: internal selection of contact-line dynamics, Physical Review Letters 115 (2015) 034502.

[38] D. J. Korteweg, Sur la forme que prennent les équations du mouvement des fluides si lon tient compte des forces capillaires causées par des variations de densité considérables mais continues et sur la théorie de la capillarité dans lhypothese dune variation continue de la densité, Archives Néerlandaises des Sciences exactes et naturelles 6 (1) (1901) 6.

[39] J. Dunn, J. Serrin, On the thermomechanics of interstitial working, Archive for Rational Mechanics and Analysis 88 (2) (1985) 95-133.

[40] D. Diehl, Higher order schemes for simulation of compressible liquidvapor flows with phase change, Ph.D. thesis, Albert-Ludwigs-Universitat (2007).

[41] J. Liu, C. M. Landis, H. Gomez, T. J. Hughes, Liquid-vapor phase transition: Thermomechanical theory, entropy stable numerical formulation, and boiling simulations, Computer Methods in Applied Mechanics and Engineering 297 (2015) 476-553.

[42] J. Bueno, C. Bona-Casas, Y. Bazilevs, H. Gomez, Interaction of complex fluids and solids: Theory, algorithms and application to phase-changedriven implosion, Computational Mechanics 55 (6) (2015) 1105-1118.

[43] Y. Bazilevs, V. Calo, T. Hughes, Y. Zhang, Isogeometric Fluid-Structure Interaction: Theory, algorithms, and computations, Computational Mechanics 43 (1) (2008) 3-37.

[44] T. Hughes, J. Cottrell, Y. Bazilevs, Isogeometric Analysis: CAD, Finite Elements, NURBS, exact geometry and mesh refinement, Computer Methods in Applied Mechanics and Engineering 194 (39-41) (2005) 4135 -4195 .

[45] J. Chung, G. Hulbert, A time integration algorithm for structural dynamics with improved numerical dissipation: The generalized- $\alpha$ method., Journal of Applied Mechanics 60 (1993) 371-375.

[46] H. Gomez, T. Hughes, X. Nogueira, V. Calo, Isogeometric Analysis of the isothermal Navier-Stokes-Korteweg equations, Computer Methods in Applied Mechanics and Engineering 199 (25-28) (2010) 1828-1840. 